FORMATION Formation emploi

Revue française de sciences sociales

143 | Juillet-Septembre 2018

Le retour en formation : une vraie chance?

\title{
La « deuxième chance " : ce que les jeunes sont capables d'en saisir
}

"Second chance" : what young adults are able to understand

Die zweite Chance und die Fähigkeit der Jugendlichen, diese wahrzunehmen

La "segunda oportunidad" : lo que los jóvenes son capaces de captar

\section{Maël Loquais et Isabelle Houot}

\section{(2) OpenEdition}

Journals

\section{Édition électronique}

URL : http://journals.openedition.org/formationemploi/6170

DOI : 10.4000/formationemploi.6170

ISSN : 2107-0946

\section{Éditeur}

La Documentation française

\section{Édition imprimée}

Date de publication : 20 novembre 2018

Pagination : 79-97

ISSN : 0759-6340

\section{Référence électronique}

Maël Loquais et Isabelle Houot, « La « deuxième chance » : ce que les jeunes sont capables d'en saisir », Formation emploi [En ligne], 143 | Juillet-Septembre 2018, mis en ligne le 20 novembre 2020 consulté le 05 novembre 2020. URL : http://journals.openedition.org/formationemploi/6170 ; DOI : https://doi.org/10.4000/formationemploi.6170 


\title{
La « deuxième chance »: ce que les jeunes sont capables d'en saisir
}

\begin{abstract}
MAËL LOQUAIS
Maître de conférences à l'université Rennes 2, au sein du laboratoire CREAD (Centre de recherche sur l'éducation, les apprentissages et la didactique).

Ses travaux de recherche questionnent la finalité de "deuxième chance » des dispositifs d'accompagnement, en caractérisant le rapport à la formation d'adultes engagés dans un parcours de formation ou d'insertion

ISABELLE HOUOT Maître de conférences, co-responsable de l'équipe ATIP - Activité Travail Identité Professionnelle - du LISEC (Laboratoire interuniversitaire en sciences de l'éducation et de la communication). Ses principaux travaux visent à caractériser et questionner les évolutions des dispositifs dits de «formation des adultes » à l'université et dans le champ de la formation et de l'insertion
\end{abstract}

Résumé

La « deuxième chance » : ce que les jeunes sont capables d'en saisir

Cette recherche porte sur ce que des jeunes en École de la deuxième chance (E2C) perçoivent de l'offre de « deuxième chance » qui leur est proposée, à partir d'un travail de thèse portant sur le terrain des E2C. Mobilisant un corpus de 40 entretiens de jeunes inscrits dans ces écoles, l'analyse porte sur les modes d'engagement dans un tel dispositif. Si les résultats signalent de fortes inégalités à l'intérieur même d'un dispositif tel qu'E2C, ils prennent appui sur l'hypothèse que les jeunes sont capables d'analyser les enjeux liés à la construction de leur propre trajectoire.

Mots clés : représentation de la formation, mesure jeune, accompagnement en formation, accompagnement professionnel, école de la deuxième chance, jeune en difficulté

Abstract

\footnotetext{
"Second chance" : what young adults are able to understand
}

This research is about how young adults understand second chance. Based on 40 interviews of young adults in second chance schools (E2C), the analysis reveals several forms of training commitment. If results show inequalities within E2C, they are based on the hypothesis that young people in $\mathrm{E} 2 \mathrm{C}$ are able to analyze issues which concern them.

Keywords: perception of training, youth employment schem, coaching in training, coaching, second-chance school, young person in difficulty

Journal of Economic Literature: I 38 ; $\mathbf{~} 68$

Traduction : Auteur-e-s. 


\section{Introduction}

L'offre de deuxième chance s'inscrit aux confluents de logiques contradictoires alliant libéralisation de l'économie et prise en compte des publics les plus fragiles. Les politiques d'insertion menées à destination des publics dits "en difficultés ", depuis plus de 40 ans, attestent d'intentions éducatives relevant de la lutte contre le décrochage et l'échec scolaire, contre les exclusions (et notamment le chômage des jeunes). Les fluctuations qui animent ces politiques à l'échelon national ou régional impactent directement les publics dits "sans qualifications "; certaines périodes sont ainsi caractérisées par une offre moins diversifiée, ce qui influe sur leurs possibilités effectives d'engagement dans la formation et/ou dans la construction d'un projet professionnel. À l'échelon européen, l'ambition consiste à "favoriser des dispositifs de deuxième chance [en vue de palier les] difficultés de réinsertion sociales des jeunes et adultes concernés" (Commission européenne, 1995, p. 23). Initiée par l'Europe dans une telle perspective, l'École de la deuxième chance vise l'insertion professionnelle et sociale des jeunes de 16 à 25 ans, sortis du système scolaire sans diplôme.

Engagés dans une formation en alternance qui peut durer sept mois, les stagiaires suivent des cours de remise à niveau, font des stages en entreprise, sont accompagnés dans la construction d'un projet professionnel et participent à des projets collectifs. Le projet professionnel, le portefeuille de compétences, les techniques de recherche d'emploi constituent autant d'éléments clés dans l'offre de formation, pour des publics qui ont le plus souvent connu un rapport difficile à l'école « de la première chance ».

De fait, s'il y a une "deuxième chance ", c'est qu'à l'évidence, une première a existé et qu'elle n'a pas fonctionné. Or, les phénomènes de reproduction touchent aussi bien l'école "de la première chance ", au sein de laquelle les inégalités scolaires, corrélées avec les inégalités sociales, ont tendance à se durcir depuis les années 2000 (Felouzis, 2012), que la formation pour les adultes où ce sont globalement les plus formés qui bénéficient de la "deuxième chance " (Voisin, 2011, p. 46). Par ailleurs, le don de deuxième chance est porteur d'une charge d'implicite : l'impératif de ne pas laisser passer sa chance. Or, si les injonctions au projet se sont généralisées à toutes les catégories professionnelles, elles touchent plus particulièrement les publics les plus précarisés. Ce paradoxe interroge la capacité d'un dispositif de "deuxième chance » à infléchir les trajectoires et aussi la façon dont les jeunes composent avec ces injonctions au projet.

Ces différents éléments amènent à considérer la " deuxième chance " au regard des injonctions implicites qui la traversent. En effet, qui refuserait une deuxième chance, et au nom de quoi ? Dans cette perspective, les dispositifs dits "de deuxième chance " constituent un analyseur singulier de ce qui se joue plus globalement dans les rapports sociaux autour de la " mise au travail » des "publics précaires ». La présente contribution vise à interroger le dispositif de deuxième chance car il participe à l'élaboration 
de normes autour du rapport au travail et à la formation. Partant d'un travail de thèse réalisé (Loquais, 2016) sur le terrain des E2C, il nous semble intéressant de convoquer ce que les jeunes bénéficiaires saisissent d'un dispositif de ce type. Comment perçoivent-ils les injonctions implicites relatives à la « deuxième chance » qui leur est proposée ? Comment en saisissent-ils les ouvertures possibles et les opportunités ? Nous questionnons ici le rapport à la deuxième chance de jeunes engagés dans un dispositif tel qu'E2C, en nous focalisant sur le sens qu'ils accordent à leur formation.

À la suite d'Hirschman (1995), on peut considérer que les stagiaires jaugent leur engagement au regard de l'alternative de choix qui s'offre à eux dans le cas où l'offre ne correspond pas à leurs attentes, entre "défection " (ici entendue comme perspective d'insertion alternative au dispositif) et " prise de parole " (définie comme forme d'expression du mécontentement à l'égard du dispositif). Le dispositif est ici abordé en tant qu'il constitue un "entre-deux» (Peeters et Charlier, 1999) caractérisant un projet sur autrui potentiellement injonctif qui n'en détermine pas pour autant les modes d'appropriation. Car si c'est au cœur de l'expérience individuelle que se cristallisent les tensions du social (Dubet, 2009 ; Martuccelli, 2010), il paraît déterminant de questionner précisément ce qui fonde les motifs d'engagement. En cela, le dispositif E2C est appréhendé en tant que support d'un projet de " deuxième chance " dont l'issue dépend moins des intentions initiales que de l'effectivité de ses usages.

Nous intéressant ici au rapport à la " deuxième chance ", nous nous appuyons sur les élaborations discursives produites par ces jeunes en situation d'entretien, car elles constituent les traces d'une activité réflexive portant sur leur vécu (cf. encadré 1). 


\section{Encadré 1. Méthodologie}

Des entretiens ont été réalisés sur la base du volontariat. Il était particulièrement important que les jeunes puissent être dans une situation suffisamment ouverte pour qu'ils s'y expriment librement. C'est pourquoi le chercheur s'est déplacé à plusieurs reprises pour rencontrer les jeunes. La situation d'entretien offre ainsi une occasion d'adressage à autrui, au travers de laquelle le sujet met en récit ses motifs d'engagement au regard de ce qu'il saisit (au sens actionnel et cognitif du terme) de l'offre de " deuxième chance ». C'est cette activité de communication vers autrui qui intéresse le chercheur, puisqu'elle révèle la façon dont l'interviewé se positionne au regard du dispositif et de ses engagements propres. Le questionnement portait sur le sens que ces jeunes attribuent à leur formation, à ses finalités et ses apports ou freins en termes d'apprentissages dans leur parcours. 40 entretiens ont été réalisés auprès de jeunes âgés de 18 à 25 ans ( 19 femmes et 21 hommes). Si la répartition du genre correspond à celle que l'on retrouve à l'échelle nationale, il n'en va pas de même du niveau scolaire : notre corpus fait état d'un tiers de diplômés, alors qu'ils représentent moins de $10 \%$ au niveau national (DARES, 2014) (*).

Le traitement des données, centré sur l'activité discursive des jeunes (à savoir ce qu'ils ont dit de leur engagement en École de la deuxième chance (E2C), a permis d'élaborer quatre thématiques en partant du discours des jeunes basés sur leur positionnement entre injonctions perçues et choix délibérés. "En se concentrant sur les activités plutôt que sur les gens, on se force à s'intéresser au changement plutôt qu'à la stabilité, à la notion de processus plutôt qu'à celle de structure » (Becker, 2002, p. 90).

(*) : À l'échelon national, le rapport DARES (2014) mentionne 14150 jeunes accueillis dans les E2C en 2013 , dont $88 \%$ sont sans diplôme (10 \% possèdent un CAP (Certificat d'aptitude professionnelle), $2 \%$ ont un $\mathrm{Bac})$ ). Parmi les non-diplômés, les niveaux sont très hétérogènes : $17 \%$ de sorties avant une 3è générale, $36 \%$ en première année de CAP-BEP (Brevet d'études professionnelles) - fin 3è, $29 \%$ ont un niveau V (CAP) sans diplôme et $6 \%$ ont un niveau Bac sans diplôme.

Dans une première partie, nous présentons la diversité et l'hétérogénéité des profils rencontrés dans cette recherche. Dans un deuxième temps, nous revenons sur les types de discours élaborés par ces jeunes. En conclusion, nous nous appuyons sur ces résultats pour mettre en évidence différentes formes de rapport au dispositif et réinterroger les biais et les ressorts de la "deuxième chance".

\section{Des parcours hétérogènes et des configurations familiales contrastées}

La région dans laquelle le recueil de données a été mené connaît une situation économique et sociale dégradée, avec un taux de chômage plus élevé que la moyenne nationale (notamment chez les jeunes de moins de 25 ans - source INSEE). Elle est parmi les territoires les mieux dotés en E2C. 
Les $\mathrm{E} 2 \mathrm{C}$ sont portées par une structure associative et organisées en réseau à l'échelle régionale. Parmi les trois sites E2C investigués sur ce territoire, l'un se situe dans une ville phare de la métropole, en périphérie, avec une forte capacité d'accueil (près de 200 stagiaires à l'année). Les deux autres sites sont situés dans des zones d'activités de villes de taille plus modeste (entre 15 et 20000 habitants), appartenant à des communautés de communes drainant de nombreuses communes rurales.

La période à laquelle ont été menés les entretiens (de janvier à avril 2014) était marquée par une offre d'insertion peu diversifiée, l'E2C faisait alors figure de passage quasi obligé pour toute reprise de formation dans une perspective d'insertion socioprofessionnelle. Ce fut moins le cas par la suite, avec le déploiement de la "Garantie Jeunes ${ }^{1}$ ", à partir de l'année suivante. Face à des difficultés d'accès à l'emploi et à l'expérience réitérée de refus d'embauche, ces jeunes perçoivent le passage en E2C comme quasi obligé pour accéder à l'emploi ${ }^{2}$. Ces tendances générales masquent cependant une forte hétérogénéité des parcours et des configurations sociales et familiales.

\subsection{Les parcours de formation et professionnels}

Notre échantillon comprend des diplômés parmi les sondés. En effet, sur les quarante jeunes interviewés, treize possèdent des diplômes (cinq $\mathrm{CAP}^{3}$, trois $\mathrm{BEP}^{4}$, quatre $\mathrm{Bac}$ professionnels, un Bac général), soit le tiers du corpus. La quasi-totalité (douze sur les treize jeunes concernés) a obtenu un diplôme par le biais d'un contrat en alternance. Deux ont choisi l'E2C pour changer d'orientation, alors qu'ils avaient une expérience dans la chaudronnerie et la soudure. La personne titulaire d'un Bac général, qui a obtenu le statut de demandeur d'asile, a suivi trois années universitaires en faculté de droit en France (en échouant à trois reprises pour la validation de la première année).

Parmi les vingt-sept jeunes "sans qualification ", les parcours de formation sont fortement différenciés. Outre le niveau et les filières suivies, les modalités de sortie du système scolaire constituent les lignes de partage entre les types de trajectoires. L'interruption du parcours est liée, pour sept d'entre eux, à une rupture du contrat d'alternance (quatre CAP, trois Bac Pro). Ce sont le plus souvent des difficultés relationnelles avec les employeurs ou tuteurs de stages qui sont évoquées pour expliquer

1. Le dispositif « Garantie jeunes », piloté par le ministère de l’Emploi, via les Missions locales, est un dispositif d'insertion destiné aux jeunes de 18-25 ans pas ou peu diplômés.

2. Tous les interviewés ont déclaré que leur inscription à E2C relevait d'un choix plus ou moins contraint du fait de l'absence d'options alternatives sérieuses.

3. Certificat d'aptitude professionnelle.

4. Brevet d'études professionnelles. 
ces situations de rupture. Quatre jeunes ont des parcours liés à des dispositifs d'adaptation ou d'insertion (type SEGPA 5 ou DAIP ${ }^{6}$ ).

Un quart des jeunes interrogés, soit quatorze personnes, ont quitté le collège en quatrième ou en troisième. Cette proportion est assez proche des moyennes nationales ( $17 \%$ de sorties avant la troisième générale pour les $\left.\mathrm{E}^{2} \mathrm{C}^{7}\right)$. Ces jeunes ont rencontré des difficultés scolaires et relatent des problèmes relationnels par rapport aux attendus de l'école, qui font écho à des ruptures familiales (alcoolisme des parents, placement en foyer...). Enfin, deux jeunes ont un parcours particulier. L'un a interrompu brutalement sa scolarité en première technologique, alors qu'il avait de bons résultats scolaires. L'autre jeune, primo arrivant, a échoué à l'examen du CAP en Afrique.

La quasi-totalité des expériences professionnelles (reconnues comme telles) mentionnées dans les entretiens concernent les périodes d'apprentissage en alternance. Les jeunes hommes qui ne sont pas passés par l'apprentissage font état de périodes d' " inactivité » marquées par des activités peu valorisées socialement (jeux vidéo en réseau), du travail "au noir ", voire des activités illicites (vente de produits stupéfiants, vols). Les jeunes femmes rapportent des expériences non déclarées de prise en charge d'un proche (parent, nièce), qui peuvent constituer des activités occupant près d'un temps plein.

\subsection{Des ancrages familiaux déterminants}

Les trois-quarts des jeunes interviewés (vingt-huit sur quarante) sont logés par un (ou leurs deux) parent(s). Toutefois, les ancrages familiaux varient suivant la façon dont s'organisent les solidarités dans la famille. Vivre chez ses parents peut générer des relations conflictuelles entre certains jeunes et leurs parents (éléments relatés dans six entretiens). Les ruptures scolaires sont souvent concomitantes à des ruptures familiales. Les situations familiales diffèrent suivant la présence ou non des deux parents et en fonction du genre.

En effet, les femmes, en particulier lorsqu'elles vivent chez un parent seul, sont sollicitées pour les tâches domestiques. Deux d'entre elles ont eu la charge d'un de leurs parents gravement malade (handicap physique pour l'un, maladie psychique à tendance suicidaire pour l'autre) pendant plusieurs années, nécessitant une présence et une activité proche du plein temps. Trois jeunes femmes sont mères de famille. Elles ont la particularité de vivre sous le toit parental. Une des femmes qui vivait seule est

5. Section d'enseignement général et professionnel adapté.

6. Dispositif d'aide à l'insertion professionnelle.

7. Source : rapport DARES, 2014. 
retournée au domicile maternel à la naissance de son enfant. De même, une autre femme a dû revenir vivre chez sa mère pour pouvoir suivre la formation E2C.

De plus, les solidarités familiales constituent un ressort important pour bénéficier d'une aide pour le transport sur les lieux de formation, ou pour financer le permis de conduire, notamment pour les jeunes qui vivent dans des communes rurales mal desservies en transports collectifs. C'est le cas d'une des jeunes diplômées qui déclare avoir pu suivre sa formation en apprentissage grâce à la disponibilité de ses parents :

"Moi quand j'étais en apprentissage, mon père, il m'emmenait tous les jours à 5 heures du matin. Donc euh... j'ai des parents qui me suivent quoi. C'est bien... il faut hein " (Olivia, 22 ans, titulaire d'un CAP vente).

Le suivi de filières d'insertion pour raison de santé (pouvant aller jusqu'à la reconnaissance d'un handicap, notamment pour des cas d'épilepsie - deux entretiens) a également été possible grâce à un certain soutien parental. D'autres parcours sont singuliers : une femme demandeur d'asile en quête d'un emploi qui permettrait de stabiliser sa situation administrative ; deux jeunes primo arrivants, soutenus financièrement par leurs parents pour suivre la formation E2C en France.

S'agissant des douze ${ }^{8}$ jeunes restant, soit un quart de notre panel, trois sont accueillis dans la famille proche (l'un est accueilli en couple chez ses beaux-parents, les deux autres chez les grands-parents ou la sœur), deux sont en foyer de jeunes travailleurs (après placement en foyer pour l'une, et exclusion du domicile paternel pour l'autre), un jeune est incarcéré la nuit, quatre vivent seuls en appartement. L'un d'eux est en procédure judiciaire contre sa mère. L'absence de soutien parental constitue un point commun à ces différents parcours.

\section{Du côté des discours: ce que les jeunes saisissent de la « deuxième chance »}

Les discours traduisent des formes d'élaborations que les jeunes produisent à partir de leur vécu et communiquent en situation. Un double niveau d'analyse peut être appréhendé pour capter ce qui se joue autour de ces élaborations. D’une part, ce qu'ils confèrent comme sens à leur engagement à E2C (et à la " deuxième chance " qui leur est "donnée ") et ce qu'ils affichent et "mettent en intrigue " (Ricoeur, 1983) en situation d'entretien. Cette dernière, en tant que tentative de communication de son vécu à autrui, constitue une activité critique. En témoigne, par exemple, cette stagiaire qui

8. Pour deux d'entre eux, nous n'avons pas d'éléments sur la situation sociale et familiale. 
questionne les termes « école » et " deuxième chance » au regard de ce qu' elle en perçoit en termes de développement professionnel :

"Donc, c'est pour ça que ça c'est bien le truc de deuxième chance. Moi, ça me permet d'avoir une deuxième chance (...) Après 'école', je trouve qu'il est pas trop bien le mot là pour ici, parce que c'est pas trop une école, c'est plus euh... Si, y a de la remise à niveau, mais je veux dire, on travaille plus sur le professionnel. C'est pas comme si c'était le collège où... là on est vraiment sur le travail, tout ça» (Véronique, 19 ans, vit seule en appartement).

Le tableau 1 reprend les résultats de la recherche en associant les types de discours aux profils des jeunes concernés. Dans cette partie, nous revenons sur quatre modes d'engagement qui ont pu être repérés dans les discours :

- L'émancipation (quinze entretiens) : valorisation d'un investissement dans la formation qui s'inscrit dans une certaine continuité avec la formation ;

- L'ambivalence (treize entretiens) : tentative de distinction par rapport à son parcours antérieur pour se conformer aux attendus d'E2C;

- L'opposition (trois entretiens) : mise en avant de désaccords avec l'accompagnement proposé en E2C;

- Le retrait (neuf entretiens) : affichage d'un certain désintérêt pour les activités du dispositif au regard des problèmes à résoudre en dehors de la formation. 
Tableau 1. Discours produits au regard des types de profils

\begin{tabular}{|c|c|c|c|c|c|}
\hline & & Emancipation & Ambivalence & Opposition & Retrait \\
\hline \multirow{4}{*}{ 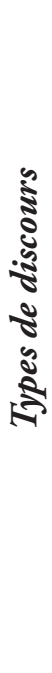 } & $\begin{array}{l}\text { Engagement dans } \\
\text { la formation }\end{array}$ & $\begin{array}{l}\text { Engagement dans toutes } \\
\text { les activités E2C qui } \\
\text { prennent sens dans un } \\
\text { parcours de vie }\end{array}$ & $\begin{array}{l}\text { Engagement dans toutes } \\
\text { les activités (dont celles } \\
\text { qui ne font pas sens). }\end{array}$ & $\begin{array}{c}\text { Désaccord avec les forma- } \\
\text { teurs sur les projets à } \\
\text { prioriser }\end{array}$ & $\begin{array}{l}\text { Désinvestissement de la } \\
\text { formation E2C }\end{array}$ \\
\hline & $\begin{array}{l}\text { Engagement dans } \\
\text { le monde }\end{array}$ & $\begin{array}{l}\text { Parcours dans une } \\
\text { logique de continuité } \\
\text { entre vécu hors E2C et } \\
\text { activités E2C ; accomplis- } \\
\text { sement de soi }\end{array}$ & $\begin{array}{l}\text { Distinction d'avec son } \\
\text { passé }\end{array}$ & $\begin{array}{l}\text { Engagements dans la vie } \\
\text { d'adulte (responsabilités } \\
\text { assumées non reconnues par } \\
\text { les formateurs E2C) }\end{array}$ & $\begin{array}{c}\text { Difficultés insolubles dans } \\
\text { la vie quotidienne liées à } \\
\text { la survie }\end{array}$ \\
\hline & $\begin{array}{l}\text { Perception du } \\
\text { dispositifE2C }\end{array}$ & $\begin{array}{c}\text { Sentiment d'être en } \\
\text { phase avec les finalités, } \\
\text { les valeurs, les modalités } \\
\text { d'accompagnement }\end{array}$ & $\begin{array}{l}\text { Promotion d'E2C; } \\
\text { distinction d'avec les } \\
\text { stigmates véhiculés } \\
\text { sur E2C }\end{array}$ & $\begin{array}{l}\text { E2C vu comme un déclasse- } \\
\text { ment (formation associée } \\
\text { à l'école) }\end{array}$ & $\begin{array}{l}\text { E2C vu comme une impasse } \\
\text { et/ou une aide inadaptée }\end{array}$ \\
\hline & Perception de soi & $\begin{array}{c}\text { Valorisation de soi } \\
\text { confirmée par le regard } \\
\text { d'autrui (figure familiale, } \\
\text { formateur, pairs...) }\end{array}$ & $\begin{array}{l}\text { Valorisation de soi ; } \\
\text { distinction d'avec le } \\
\text { regard infamant d'autrui }\end{array}$ & $\begin{array}{c}\text { Valorisation de soi en dehors } \\
\text { d'E2C ; dévalorisation de soi } \\
\text { dans E2C }\end{array}$ & Dévalorisation de soi \\
\hline \multirow{3}{*}{ 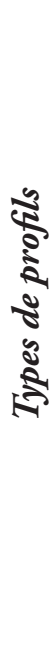 } & Genre & $\begin{array}{l}9 \text { femmes } \\
6 \text { hommes }\end{array}$ & $\begin{array}{l}10 \text { hommes } \\
3 \text { femmes }\end{array}$ & 3 femmes & $\begin{array}{l}5 \text { hommes } \\
4 \text { femmes }\end{array}$ \\
\hline & $\begin{array}{l}\text { Situation sociale } \\
\text { et familiale }\end{array}$ & $\begin{array}{l}14 \text { jeunes chez leurs } \\
\text { parents } \\
1 \text { jeune seul }\end{array}$ & $\begin{array}{c}9 \text { jeunes chez parents } \\
\text { séparés } \\
3 \text { jeunes chez leurs deux } \\
\text { parents } \\
1 \text { jeune en foyer }\end{array}$ & $\begin{array}{c}1 \text { mère d'un enfant } \\
1 \text { jeune prend en charge son } \\
\text { père malade } \\
1 \text { jeune sollicitée pour la } \\
\text { prise en charge de sa fratrie }\end{array}$ & $\begin{array}{c}5 \text { jeunes logés chez un } \\
\text { parent } \\
1 \text { jeune seule } \\
2 \text { jeunes en foyer } \\
1 \text { jeune incarcéré }\end{array}$ \\
\hline & Situation scolaire & $\begin{array}{c}10 \text { diplômés } \\
3 \text { jeunes niveau collège } \\
\text { (dont } 2 \text { primo arrivants) } \\
1 \text { SEGPA } \\
1 \text { jeune niveau lycée }\end{array}$ & $\begin{array}{c}8 \text { jeunes niveau collège } \\
4 \text { jeunes SEGPA ou DIMA } \\
1 \text { diplômé (CAP) }\end{array}$ & $\begin{array}{l}2 \text { diplômées } \\
1 \text { DAIP }\end{array}$ & 9 jeunes niveau collège \\
\hline
\end{tabular}

Sigles : DIMA : Dispositif d'Insertion aux Métiers d'Alternance. Segpa : Section d'enseignement général et professionnel adapté. DAIP : Dispositif d'aide à l'insertion professionnelle.

Source : Thèse de doctorat (Loquais, 2016)

\subsection{L'émancipation}

Dans quinze entretiens, les jeunes présentent leur parcours en E2C comme un tremplin d'évolution personnelle. Quels que soient les domaines (stages, activités culturelles, cours en centre, accompagnement du projet), ils souscrivent aux activités auxquelles ils parti- 
cipent. Ils disent adhérer non seulement aux contenus abordés, mais également à la pédagogie employée, basée sur une sollicitation de l'autonomie des personnes :

"Euh, ils nous intègrent un peu avant le travail, enfin avant... je sais pas comment expliquer... (...) Ils nous aident à ce que ce soit nous-mêmes, que ce soit personnel. C'est mieux quand ça vient de soi que des autres, alors que quand on est ici, ben, ils nous mettent dans le bain quoi...» (Véronique, 19 ans, vit seule).

La thématique de l'émancipation domine dans tous ces entretiens, où E2C apparaît dans une continuité assumée avec le parcours antérieur. Les jeunes insistent sur la chance qui leur est donnée de suivre cette formation qui correspond à leurs attentes et qui ouvre des possibles pour la suite de leur parcours. Tel est le cas de cette stagiaire qui évoque la « deuxième chance » comme une réelle opportunité :

"Pis du coup, maintenant, euh, j'arrive à un certain âge où je peux plus me permettre de laisser passer mes chances il faut dire. (...) C'est une chance d'être là ; je me rends compte que c'est une chance de découvrir des métiers, de concrétiser ce que je veux faire; c'est quand même une chance. Parce que qui m'aurait permis d'aller voir un métier?" (Yacina, 22 ans, titulaire d'un CAP vente).

Les grands principes d'E2C sont abordés comme entrant en résonnance avec les valeurs portées par les jeunes. La dimension humaniste du projet E2C est ici soulignée par une jeune, par ailleurs militante dans un parti de gauche :

"Ben je trouve que c'est quand même un peu gauchiste, le fait d'aider les jeunes. C'est quand même un peu de gauche de... Après, je pense que si quelqu'un vote FN ici, et qu'il s'occupe des jeunes, ce serait un peu bizarre, parce qu'ily en a quand même beaucoup qui ont des origines " (Alissia, 23 ans, CAP broderie, activités artistiques).

La confiance accordée par les formateurs est particulièrement mise en avant. Le fait de voir des choix personnels confirmés par autrui constitue un des atouts du dispositif. Les jeunes attribuent alors à ce dernier une fonction palliative au regard de leur vécu antérieur. Le fait d'être incité à mener seul des activités qu'ils n'auraient pas réalisées sans ce cadre d'exigences constitue un aspect stimulant de la formation :

"Ils nous laissent vraiment dire ce quion a envie de faire ; pareil, ils nous font confiance" (Floriane, 20 ans, titulaire d'un CAP couture).

"Ici on me pousse à le faire, sur le coup, je me dis ils sont chiants, mais bon, je sais que c'est pour mon bien quand même (...) Ma mère... notre mère nous a jamais emmenés chez le médecin, on savait pas c'était quoi limite... (Rires) c'est difficile à expliquer... ben déjà (j'ai jamais été) encadré par des parents (...) moi j’ai jamais eu de jeunesse quoi. J'avais beaucoup trop à faire pour mamuser..." (Stéphane, 21 ans, études interrompues suite à un différend familial).

Les jeunes qui développent ce type de discours habitent chez leurs parents (sauf un) et dix sont diplômés. Les cinq jeunes non diplômés ont des parcours particuliers : deux ont 
interrompu un parcours de formation contre leur gré (dans le sport et en lycée technique), deux sont primo arrivants et affichent leur motivation à s'intégrer dans leur nouvelle vie professionnelle, et un a suivi un cursus SEGPA du fait d'un handicap visuel, tout en étant accompagné de près par ses parents. Ces jeunes mentionnent volontiers l'aide dont ils ont bénéficié de la part de leur entourage proche pour construire leur parcours, comme cette jeune qui a "galéré » (selon ses termes) pour obtenir son bac:

"Ouais, je suis allée au bout de mon Bac couture. Je suis fière de moi (rires). Oui quand même, parce que je voulais arrêter, pis on m'a poussée, on m'a poussée pour que j'y aille, mon copain, ma famille. " (Caroline, 22 ans, titulaire d'un Bac couture)

Le fait d'avoir eu un parcours scolaire choisi et assumé, ayant abouti à l'obtention d'un diplôme pour dix d'entre eux, semble constituer un facteur décisif par rapport à ce que les jeunes saisissent d'un dispositif de " deuxième chance ». En effet, leur parcours scolaire est marqué par une certaine réussite, puisque l'obtention d'un diplôme à l'école « de la première chance " leur permet de bénéficier d'une reconnaissance sociale qui a eu des effets vertueux en termes d'engagement dans leurs cursus de formation ultérieurs. De plus, tous mettent en avant le rôle du soutien familial (matériel, affectif) pour bénéficier pleinement des apports de la formation. Ces données laissent penser que la perception d'E2C comme occasion d'une réelle deuxième chance demande certains acquis antérieurs à la formation.

Les discours sont en phase avec les valeurs véhiculées par E2C. À ce titre, les jeunes "émancipés» effectuent un certain lissage de toute "prise de parole (Hirschman, op. cit.), pouvant avoir des connotations critiques. Ainsi, quand bien même certains aspects sont mal vécus, un consensus s'établit sur les finalités visées du dispositif?. En cela, les sorties envisagées du dispositif sont présentées comme "positives" par les jeunes eux-mêmes. En effet, dans la mesure où ils bénéficient tous d'une culture scolaire conséquente, ils en perçoivent les codes implicites (la valorisation de l'autonomie, des projets personnels et professionnels...).

En se situant en phase avec la "norme d'internalité » (Dubois, 2009) et les attentes en termes d'autonomie qui leur sont formulées, ces jeunes parviennent à gagner la confiance des formateurs. Ils se situent dans un rapport de congruence entre le projet du formateur sur soi et le "projet de soi pour soi " (Kaddouri, 2011), dans la mesure où E2C y est perçu comme un levier d'infléchissement de leur trajectoire.

\subsection{L'ambivalence}

Un tiers des jeunes (treize entretiens) valorisent E2C en insistant sur ce qu'ils mettent en œuvre dans leur comportement pour répondre aux attendus des formateurs. Même lorsque la formation ne leur paraît pas faire sens, ils en valorisent les modalités et se conforment à

9. "Ils sont chiants, mais bon, je sais que c'est pour mon bien quand même» (Stéphane, 21 ans, vit seul). 
ce que les formateurs attendent d'eux. Il s'agit, dès l'entrée du dispositif, lors de l'entretien de recrutement ou par le suivi individuel du formateur, de faire la preuve de sa motivation à suivre la formation, ce qui pousse certains jeunes à « forcer le trait » de leur implication dans la formation :

"Sur le coup, j'ai... je me suis montré plus motivé que j'étais, forcément. Et au début, quand je suis rentré, je me suis dit (pffou), j'étais motivé, mais j'espère que ça va me plaire " (Bernard, 20 ans, a suivi un dispositif d'insertion aux métiers de l'alternance - DIMA).

Selon eux, E2C reste une voie marquée par le stigmate de l'échec scolaire. Ils présentent leur parcours actuel en rupture par rapport à un passé scolaire ou familial décrit comme " chaotique ». Les jeunes insistent ici sur un engagement de rupture en E2C par rapport à leur propre personnalité jugée comme trop puérile et ne maîtrisant pas les enjeux de l'entrée dans la vie adulte. De plus, ils tentent de mettre à distance les stigmates colportés sur les E2C, en valorisant la formation ou en se distinguant de certains de leurs pairs.

"Ben au début, quand vous entendez parler les gens, c'est... c'est une école pour les cas soc', pour ceux qui n'ont rien... mais non, ce n'est pas ça. C'est voilà, c'est pour aider les jeunes. Peut-être qu'on a eu une chance, on l'a pas saisie, mais celle-là, faut la saisir. Faut la saisir, faut être motivé " (Jonas, 21 ans, titulaire d'un CAP vente, père d'un enfant).

Ces jeunes expriment un sentiment de pression, n'ayant d'autres possibilités que de compter sur eux-mêmes pour mener à bien leurs projets. Ils perçoivent $\mathrm{E} 2 \mathrm{C}$ comme une "dernière chance " (Jonas) alors que leur avenir est vu comme très incertain. Tel est le cas de ce stagiaire en foyer, qui insiste sur l'obligation devant laquelle il se trouve de devoir gérer sa vie de façon autonome, en se distinguant des autres stagiaires :

" J'ai vécu beaucoup de choses, après je ne raconte pas toute ma vie parce que voilà, j'ai beaucoup de vécu et je pense qu'à 21 ans, déjà, grandir sans ses parents et devoir se démerder tout seul, c'est assez difficile" (Patrick, 20 ans, vit seul - parents décédés).

Quelles que soient les situations, tous ces jeunes mettent en avant leur propre volonté de "s'en sortir» (8 entretiens), en soulignant non seulement les difficultés rencontrées, mais également les principes moraux sur lesquels ils fondent leur action (par exemple la valeur travail liée au mérite). Tel est le cas de ce jeune qui dit avoir réussi à ne pas se laisser entraîner dans un parcours de délinquance au prix d'efforts qui ont été payants pour lui :

"Oui, j'aurais pu rester dans la rue et faire de l'argent facile. (...) Après j'ai commencé à traîner, mais ici, vu que la vie active, je sais ce que c'est, et bien je me dis dans la vie, si on veut quelque chose, et bien il faut s'investir » (Nathanaël, 25 ans, originaire de Martinique).

Les jeunes se situant dans un engagement ambivalent ne sont pas diplômés (à l'exception d'un jeune père pour qui nous disposons de peu d'éléments de parcours). Il s'agit majoritairement d'hommes (dix hommes pour trois femmes). Neuf vivent chez un " parent isolé ». Quatre jeunes ont suivi des cursus d'insertion type SEGPA et ont une expérience des dispositifs d'adaptation marquée par des conflits avec les enseignants. Le 
caractère précaire des situations ne transparaît pas directement dans les discours également traversés par l'ambivalence, entre expression d'un besoin de sécurité matérielle et dissimulation de motifs motivationnels liés à sa condition matérielle :

"Je lui ai tout dit, je lui ai dit que j'avais des soucis tout, mais que je comptais sur l'E2C aussi financièrement, aussi pas que financièrement, pour m'ouvrir des portes " (Patrick, 20 ans, vit seul).

Les discours sont ici caractérisés par la distinction d'avec les pairs, le passé, les stigmates véhiculés sur E2C, le risque de déclassement... Cette mise à distance d'une possible dégradation de son image traduit des stratégies que les jeunes mettent en œuvre pour répondre aux attendus du dispositif, même lorsque cela ne fait pas sens pour eux. Le "loyalisme » (Hirschman, op. cit.) dont ils font preuve indique un rapport à la deuxième chance flirtant avec l'idée de "dernière chance " (Jonas), puisqu'en en cas de défection de la formation, les jeunes sont confrontés à la menace d'une relégation durable.

Dans cette même logique, les motifs matériels d'engagement (centrés sur les besoins en termes de rémunération) sont tus ou atténués afin d'afficher une motivation correspondant aux normes attendues. L'accent est mis sur les difficultés qu'ils rencontrent à répondre aux injonctions à l'autonomie en raison de l'absence d'aide externe. Dès lors, se faire reconnaître comme sujet capable dans et par E2C devient une priorité vitale, puisque ces jeunes ne bénéficient pas d'étayages autres que ceux apportés par les formateurs. Ces éléments tendent à confirmer le caractère discriminant des injonctions au projet (Coquelle, 1994 ; Castra, 2003) en montrant qu'à l'intérieur d'un même dispositif, les dispositions des sujets à s'y engager sont inégales (suivant les étayages dont ils peuvent ou non bénéficier).

\subsection{L'opposition assumée au dispositif}

Trois jeunes décrivent l'E2C comme défaillante dans ses modalités d'accompagnement et dans les perspectives d'insertion perçues après leur passage en formation. Il s'agit de femmes dont deux sont diplômées. Elles ont la particularité d'être fortement investies dans des responsabilités familiales : l'une est mère d'un enfant, une autre a prodigué des soins à son père malade pendant plus de deux ans, la troisième est sollicitée par sa mère pour la prise en charge de la fratrie.

Pour ces femmes, E2C reste associé à ce qu'elles identifient comme appartenant au parcours de leurs propres enfants, ou frères et sœurs. Elles ont le sentiment que l'expérience de vie qu'a constitué, pour elles, la prise en charge de tierces personnes de leur famille, n'est pas valorisée par les formateurs et les responsables E2C.

"Quand on se retrouve dans... dans le contexte comme ça de l'école ben (soupir), c'est pas pareil quà la maison on va dire, on se laisse plus, euh... on se laisse plus aller, voilà, c'est plus strict à la maison, il faut montrer le modèle ; on est le modèle hein " (Mounia, 20 ans, mère d'un enfant). 
Elles construisent un discours critique vis-à-vis de l'école, qu'elles perçoivent comme un frein pour leur développement professionnel et personnel. Elles assument les désaccords qu'elles vivent avec les formateurs et défendent un point de vue contraire au leur. L'engagement à $\mathrm{E} 2 \mathrm{C}$ est vécu comme un déclassement, car les activités proposées sont jugées infantilisantes, et d'un niveau trop faible pour qu'il offre de réelles possibilités de progression. Elles n'hésitent pas à entrer en conflit avec les formateurs en défendant un projet professionnel qui ne correspond pas toujours aux propositions qui leurs sont faites :

"Ben du coup, elle (la formatrice) veut que je retourne en cantine, mais moi, je ne veux pas, encore moins en restauration, servir les clients, non, ça ne me plait pas. (...) Elle m'a dit "vous vous éloignez trop de vos projets, vous n'avez aucun projet" et tout ça, alors qu'ici on est là pour faire un projet, donc ça veut dire ce que ça veut dire, du coup, je ne sais pas, mais jirai quand même chez XXX, même si ça ne lui plait pas» (Frédérique, 25 ans, titulaire d'un CAP maintenance hygiène, a pris en charge des membres de sa famille).

La condition de stagiaire en entreprise est également mise en cause. Le sentiment d'être relégué à des tâches subalternes pour un travail qui ne donnera pas lieu à rémunération génère une incompréhension quant aux règles du jeu qui encadrent leur présence en stage. Tel est le cas de cette femme qui assume le fait d'avoir un point de vue dissonant par rapport à sa tutrice de stage quant aux attendus du travail :

"... alors 197 habits pliés et rangés dans la journée, c’est déjà pas mal. Donc du coup, je lui ai dit. Parce que quand j'ai quelque chose à dire, je le dis quoi " (Frédérique).

De plus, le refus de se conformer aux attendus s'inscrit dans des rapports de domination qu'elles perçoivent comme tels. Ainsi, en n'acceptant pas de s'engager dans des métiers perçus comme subalternes, elles affichent une insoumission aux projets d'autrui sur soi :

"Ouais, quand je réponds, tout ça c'est pour, euh, montrer que je suis pas leur soumis quoi. Je vais pas faire exactement ce qu'ils me disent alors que ça me plait pas. Pis voilà " (Frédérique).

Le rapport d'opposition caractérise l'expression de conflits ouverts avec les formateurs. Ce type de discours s'inscrit pleinement dans ce qu'Hirschman (1995) nomme la "prise de parole ", les désaccords constituent alors l'expression d'un mécontentement assumé et exprimé. La teneur critique des discours à l'égard d'E2C détonne dans ces trois entretiens par rapport au reste du corpus. S’il y a convergence de vues sur les finalités d'insertion en mettant en avant les bénéfices des stages en entreprise, ils dévalorisent le temps passé en centre de formation. La formation est vécue comme une réminiscence du modèle scolaire associé à l'image stigmatisante de l'enfance et de l'échec. L' " offre identitaire " (Kaddouri, 1996) de stagiaire ne correspond pas aux formes identitaires valorisées (professionnelles).

En cela, les sentiments d'injustice exprimés par ces personnes traduisent une demande de reconnaissance de leur expérience dans et par la formation. Ces femmes revendiquent, en quelque sorte, une deuxième chance qui n'est pas à la hauteur de leurs attentes dans le dispositif E2C. 


\subsection{Un discours de retrait}

Plus qu'une dévalorisation d'E2C, le discours de retrait (neuf entretiens) caractérise un désintérêt marqué pour le dispositif. Les jeunes soulignent leurs difficultés à faire face au quotidien. Le passage en E2C y est présenté comme inutile pour soi-même, voire également pour les autres. Les difficultés à s'impliquer dans les activités de la formation sont d'abord attribuées à des perturbations externes qui empêchent de s'y engager réellement :

"Encore ce matin, je suis pas allée en cours parce que ma mère, elle a fugué de chez elle. Ça fait deux jours qu'elle est pas rentrée, qu'il y a ma petite sœur, mon neveu, mon petit frère chez elle, euh, elle a fugué, voilà, ça fait deux jours que je la cherche " (Raphaëlle, 22 ans, en foyer de jeune travailleur).

Ils se dévalorisent en se présentant comme souvent absents et peu concernés par ce qui se déroule en centre. Pour certains, c'est le sentiment de honte qui domine, en se sentant jugé négativement du fait du stigmate de l'échec scolaire. D’autres se montrent découragés et incapables de mener les activités qu'on leur demande de réaliser :

"Et vraiment, quand ça commence à me gonfler, euh... que je vois que j'y arrive pas, ben c'est vrai que je baisse les bras et, euh, j'arrête » (Mounia, 20 ans, mère d'un enfant).

Si les stages sont présentés comme potentiellement plus intéressants, les expériences qu'ils relatent font état d'une communication difficile avec les tuteurs. Finalement, c'est par l'activité qu'ils mènent en dehors d'E2C qu'ils tentent de vivre, "ou plutôt survivre " (Raphaëlle). Le sentiment de perdre pied est exprimé par ce jeune qui entrevoit la possibilité qu'E2C conduise à une impasse :

"Après il y a un... comme j'en ai parlé à ma référente, donc XXX, j'ai une peur, c'est d'arriver en fin de parcours et... rien quoi! " (Dorian, 19 ans, interruption de scolarité en troisième).

Les jeunes qui se montrent en retrait par rapport au dispositif ont tous arrêté leurs études au niveau du collège (en quatrième ou en troisième). Ils connaissent des situations de précarité qui ne leur permettent pas de s'engager dans un projet sur la durée. Trois d'entre eux sont présents à l'E2C sous contrainte. En effet, deux jeunes en foyer de jeunes travailleurs (FJT) et un en mandat judiciaire voient la poursuite du versement de leur prestation logement ou de la liberté conditionnelle tributaires d'un suivi assidu de la formation à l'E2C. Leur présence en formation est due à cette injonction à s'y engager, mais ne constitue pas un choix assumé. Les six autres personnes vivent chez un parent seul : l'une a des problèmes de santé (dépression), un autre a des ennuis judiciaires et quatre vivent chez leurs parents en ayant fourni peu d'éléments sur leurs activités. Il s'agit de "désaffliés " (Castel, 1995) ayant construit des modes de rapport au monde à la limite de la légalité, où les activités menées se déroulent en dehors de toute forme d'institution et parfois même dans une certaine réclusion.

Dans le discours de retrait, les jeunes se situent en rupture avec la formation E2C. Aucune perspective d'amélioration de leur condition ne semble passer par le dispositif E2C. Deux 
raisons sont avancées : soit les attendus en E2C paraissent inatteignables (en termes de niveau, de capacité de travail...), soit ils ne correspondent pas à des possibilités priorisées.

En cela, les stratégies de défection dominent dans les discours, tandis que les perspectives d'ouvertures après E2C sont présentées comme moindres. Ces jeunes font face à un risque, celui de s'installer dans des dynamiques durables de désaffiliation (leur crainte de ne pas saisir « leur» chance en témoigne).

Plus qu'un simple problème d'insertion, c'est la difficulté à se voir reconnaître comme sujet capable qui est ici en jeu. Les conditions d'engagement dans la formation placent ces jeunes dans le paradoxe des injonctions au projet, puisqu'ils sont tenus de s'engager dans un projet de formation tout en bénéficiant de supports extrêmement fragiles pour assurer leur survie au quotidien.

\section{Conclusion}

De nombreuses études longitudinales (Zunigo, 2010 ; Bregeon, 2013 ; Denecheau, Houdeville, Mazaud, 2015) explorent les problématiques propres aux parcours des jeunes "sans qualification". Toutes mettent en évidence au moins deux éléments. D’une part, le problème à résoudre relève d'un processus long, le " décrochage " résultant de facteurs multivariés. D’autre part, elles soulignent le hiatus entre les finalités visées par les politiques publiques (la réduction des inégalités scolaires et sociales) et leurs effets difficilement mesurables et contrastés.

Les résultats de notre recherche apportent un double éclairage : du côté des usages d'un dispositif tel qu'une E2C, et du côté des conséquences qu’il est possible d'en tirer par rapport aux actions menées au nom de la deuxième chance. Ces résultats appellent au moins trois éléments de discussion, à savoir la mise en évidence de formes de rapports au dispositif différenciées, la tendance confirmée d'une deuxième chance réservée aux mieux lotis, et la question de l'effectivité de la promesse d'une deuxième chance.

Premièrement, même si ce type de dispositif n'inverse pas la tendance en termes de " désavantage scolaire " (Demeuse, Frandji, Greger, Rochex, 2011), c'est d'abord l'hétérogénéité qui caractérise le rapport à la deuxième chance. L'aspect multiforme des positionnements des jeunes par rapport à l'offre d'insertion transparaît dans les tensions qui caractérisent les stratégies de défection par rapport à la prise de parole. Si la visée d'E2C s'inscrit dans une perspective de « sortie positive » (retour à l'emploi ou à une formation qualifiante à l'issue de la formation), seul le rapport d'émancipation caractérise une "sortie » correspondant aux attendus du dispositif. À l'inverse, le rapport de désengagement caractérise des formes de défection par défaut, sans autre alternative convaincante. De plus, on peut noter que la prise de parole apparaît dans ce corpus, certes de façon minoritaire (trois femmes concernées pour le rapport d'opposition dans notre étude), mais de façon suffisamment prononcée pour éclairer les conceptions critiques construites par les jeunes en situation de formation. Cela tendrait à confirmer que le consensus autour de la visée de deuxième 
chance recouvre des " conflits de critères" (Clot, 2010) qui s'expriment difficilement de façon spontanée. D'ailleurs, la forte proportion de jeunes concernés par l'engagement ambivalent est révélatrice d'un "loyalisme» (Hirschman, op. cit.) face à la deuxième chance qui leur apparaît comme un projet somme toute légitime.

Deuxièmement, si nos résultats confirment que la " deuxième chance " bénéficie plutôt aux moins précaires d'entre les précaires (Castra, op. cit.), cette étude révèle que de fortes inégalités se manifestent à l'intérieur même d'un dispositif comme l'E2C. La présence de diplômés dans notre corpus (environs le tiers des jeunes interrogés) questionne non seulement les modes de sélection à l'entrée des dispositifs de deuxième chance, mais plus largement la précarisation grandissante d'une part importante de la jeunesse, en particulier lorsque l'offre d'insertion est peu diversifiée, comme c'était le cas au moment du recueil de données (avec l'absence d'alternatives telles que le dispositif Garantie Jeunes). Dans ce contexte, le caractère discriminant du projet est confirmé par notre étude. En effet, les publics qui font face aux plus fortes incertitudes sont les moins disposés à s'engager dans le dispositif. Pour autant, les injonctions au projet génèrent des effets fortement différenciés suivant les jeunes. Contrairement à ce qu'on pourrait en attendre, il apparaît même que les menaces perçues par les jeunes relèvent moins du dispositif en tant que tel que d'une possible relégation à l'issue de leur cursus de formation. À ce titre, les jeunes les moins étayés restent engagés dans le dispositif à partir du moment où ils analysent la défection comme l'issue la moins favorable, sans qu'ils se fassent réellement d'illusions sur les possibilités d'insertion effectives (et durables).

Enfin, ces résultats offrent une ouverture stimulante pour penser les conditions d'une deuxième chance effective. Si nos résultats confirment les capacités stratégiques que les publics dits " en difficulté» mettent en œuvre dans la formation (Plomb, Henchoz, 2014 ; Lavielle-Gutnik, 2007), ils montrent également une capacité d'analyse de leurs conditions de vie matérielle, sociale... Ici, la mise en évidence d'éléments de leur vie quotidienne et concrète qui leur permettraient d'envisager l'offre de deuxième chance comme une réelle opportunité (ou au contraire comme inaccessible) relève bien des "capabilités " (Sen, 2010), c'est-à-dire des possibilités réelles de choisir les modes d'action valorisés et priorisés par les sujets. Ainsi, l'effectivité de la deuxième chance, telle qu'elle est analysée par les jeunes, relève moins des dispositions offertes dans cette perspective que des conditions culturelles, sociales et matérielles qui surdéterminent leurs possibilités d'y accéder.

Dans un contexte où la généralisation des injonctions au projet et à l'autonomie a des effets discriminants pour les publics les plus éloignés de l'emploi et de la formation, le développement de dispositifs menés au nom du " don " de deuxième chance pose question. L'égalité des chances concerne non seulement l'offre de première et de deuxième chance, mais plus largement ce qui fonde les principes de participation à la vie sociale. En s'appuyant sur un sujet capable de participer à l'analyse du problème posé et aux voies envisagées pour y remédier, de nouvelles perspectives de co-élaboration des dispositifs de deuxième chance pourraient voir le jour. 


\section{Bibliographie}

Becker H. (2002), Les ficelles du métier, Paris, La Découverte.

Bregeon P. (2013), Parcours précaires. Enquête sur une jeunesse déqualifiée, Rennes, Presses Universitaires de Rennes.

Castel R. (1995), Métamorphoses de la question sociale. Une chronique du salariat, Paris, Librairie Arthème-Fayard.

Castra D. (2003), Linsertion professionnelle des publics précaires, Paris, Presses Universitaires de France.

Clot Y. (2010), Le travail à cour. Pour en finir avec les risques psychosociaux, Paris, La Découverte.

Commission européenne (1995), Enseigner et apprendre, vers la société cognitive. Livre blanc sur l'éducation et la formation.

Coquelle C. (1994), «Attention, projet! », Formation Emploi, 45, pp. 25-32.

DARES (2014), "L'École de la Deuxième Chance. La "grande école" des décrocheurs motivés ", Dares analyses, 68.

Demeuse M., Frandji D., Greger D., Rochex J.-Y. (dir.) (2011), Les politiques d'éducation en Europe. Tome II. Quel devenir pour l'égalité scolaire?, Lyon, ENS Éditions.

Denecheau B., Houdeville G., Mazaud C. (dir.) (2015), A l'école de l'autonomie. Épreuves et enjeux des dispositifs de deuxième chance, Paris, L'Harmattan.

Dubois N. (2009), La norme d'internalité et le libéralisme, Paris, Presses Universitaires de France.

Dubet F. (2009), Le travail des sociétés, Paris, Éditions du Seuil.

Felouzis G. (2012), «Le système éducatif français dans l'OCDE : quelles performances ?» Cahiers français, 368, pp. 9-15.

Frétigné C. (2011), Exclusion, insertion et formation en question, Paris, L'Harmattan.

Hirschman A. (1995), Défection et prise de parole, Paris, Fayard.

Kaddouri M. (2011), "Motifs identitaires des formes d'engagement en formation ", Savoirs, 25, pp. 69-86.

Kaddouri M. (1996), "Place du projet dans les dynamiques identitaires ", Éducation permanente, 128, pp. 135-151.

Lavielle-Gutnik N. (2007), Devenir soi-même comme les autres. Sens donné et engagement des sujets en autoformation : le cas des APP, Thèse de doctorat en Science de l'Éducation, Paris, CNAM. 
Loquais M. (2016), Les modes d'engagement en contexte d'injonction au projet : le cas des jeunes des Écoles de la Deuxième Chance, thèse de doctorat, sciences de l'éducation, Lille, université Lille 1.

Martuccelli D. (2010), La société singulariste, Paris, Armand Colin.

Peeters H., Charlier P. (1999), "Contributions à une théorie du dispositif ", Hermès, 25, pp. 15-23.

Plomb F., Henchoz C. (2014), «Les engagements infrapolitiques des jeunes en difficulté d'insertion : entre institutions et pratiques autonomes ", Sociétés et jeunesses en difficulté, 14. Récupéré sur : http://sejed.revues.org/7772

Ricoeur P. (1983), Temps en récit, tome I, Paris, Seuil.

Sen A. (2010), L'idée de justice, Paris, Flammarion.

Voisin A. (2011), "L'économie de la formation », in Carré P. et Caspar P. (dir.), Traité des sciences et des techniques de la formation, Paris, Dunod, pp. 43-59.

Zunigo X. (2010), « Le deuil des grands métiers. Projet professionnel et renforcement du sens des limites dans les institutions d'insertion ", Actes de la recherche en sciences sociales, 184, pp. 58-71. 